\title{
Modelo matemático para o planejamento da circulação de trens em ferrovias de linha singela
}

\author{
Edson Pimentel Pereira ${ }^{1}$, Rodrigo de Alvarenga Rosa², Pedro Pelacani Berger ${ }^{3}$, João Teixeira \\ Carvalho ${ }^{4}$ e Glaydston Mattos Ribeiro ${ }^{5}$
}

\begin{abstract}
Resumo: Nos últimos anos a exportação de commodities agrícolas e minerais vem crescendo no Brasil e isto tem levado a um aumento de demanda de transporte ferroviário. Assim, é importante que modelos matemáticos sejam propostos para o planejamento da circulação dos trens que tenham por objetivo o aumento da quantidade de trens circulando na ferrovia. Desta forma, este artigo apresenta um modelo matemático baseado no modelo Job Shop para o Problema de Planejamento da Circulação de Trens em uma ferrovia de linha singela. O modelo proposto permite que os trens sejam atrasados ou adiantados na partida visando reduzir o tempo parado em pátios de cruzamento. O modelo foi resolvido utilizando CPLEX 12.6 e testes foram realizados com dados reais da Ferrovia Centro Atlântica (FCA). Os resultados do modelo foram comparados com os resultados da FCA e em todos os cenários testados o modelo obteve redução do tempo de viagem dos trens.

Palavras-chave: problema de planejamento da circulação de trens, operação ferroviária, Train Timetabling Problem, transporte ferroviário.
\end{abstract}

\begin{abstract}
In the last years the export of agricultural and mineral commodities has increased in Brazil and this has led to an increase in rail demand to railroad transport. Then, it is important that mathematical models shall be proposed for the planning of trains' movement with the objective to increase the amount of circulating trains. Thus, this paper presents a mathematical model based on the Job Shop model to solve the Train Timetabling Problem on a single track railroad. The proposed model allows the trains to have an earlier or delayed departure time in order to reduce the stopped time at a crossing yard. The model was solved using CPLEX 12.6 and tested were done with real data of Ferrovia Centro Atlântica (FCA). The model results were compared with the results of the current planning of FCA and in all tested scenarios the model obtained a reduction of the trains travel time.
\end{abstract}

Keywords: Train Timetabling Problem, railroad operation, railroad transport.

\section{INTRODUÇÃO}

O transporte ferroviário no Brasil aumentou 133\%, passando de 137,2 bilhões de TKU (toneladas por quilômetro útil), em 1997, para 320,0 bilhões de TKU, em 2012 (CNT, 2013). No entanto, a malha ferroviária cresceu 1,29\% de 1997 a 2012 (CNT, 2013 e GEIPOT, 2001).

O planejamento dos trens que irão utilizar a linha ferroviária em certo intervalo de tempo é fundamental para utilizar ao máximo a capacidade da ferrovia. Visando contribuir com a melhoria do planejamento dos trens que irão utilizar a linha ferroviária e, consequentemente, com um possível aumento de trens circulando na mesma, este artigo apresenta um modelo matemático baseado no modelo Job Shop para o planejamento da circulação de trens em uma ferrovia de linha singela. O modelo proposto neste artigo tem por objetivo a redução do tempo total de viagem de todos os trens e, indiretamente, o modelo pode levar a uma redução do tempo parado em pátios de cruzamento de todos os trens.

\footnotetext{
${ }^{1}$ Edson Pimentel Pereira, Mestrado em Engenharia Civil - Transportes, UFES. (edson.pereira@ifes.edu.br)

2 Rodrigo de Alvarenga Rosa, Mestrado em Engenharia Civil -

Transportes, UFES. (rodrigo.a.rosa@ufes.br)

${ }^{3}$ Pedro Pelacani Berger, Mestrado em Engenharia Civil - Transportes,

UFES. (pedropberger@gmail.com)

4 João Teixeira Carvalho, Departamento de Engenharia, VLI S/A.

(joao.teixeira.carvalho@vli-logistica.com.br)

${ }^{5}$ Glaydston Mattos Ribeiro, Programa de Engenharia de Transportes,

COPPE-UFRJ. (glaydston@pet.coppe.ufrj.br)
}

Manuscrito recebido em 19/03/2015 e aprovado para publicação em 08/08/2016

Este artigo é parte de TRANSPORTES v. 25, n. 1, 2017. ISSN: 2237-1346 (online). DOI:10.14295/transportes.v25i1.903
Visando contribuir com a melhoria do planejamento de circulação de trens em ferrovias de via singela, este artigo apresenta um modelo matemático baseado no modelo Job Shop para o planejamento da circulação de trens. O modelo proposto neste artigo tem por objetivo a redução do tempo total de viagem de todos os trens e, indiretamente, o modelo leva a uma redução do tempo parado em pátios de cruzamento de todos os trens.

O modelo proposto tem duas características que o diferencia dos demais já publicados. Primeiro, ele define o horário de partida de cada trem respeitando uma janela de horário de partida definida pelo cliente do trem. Segundo, ele calcula o horário de chegada de cada trem respeitando uma janela de horário de chegada estabelecida também pelo cliente do trem. Essas duas características são importantes, pois alguns pátios ferroviários não operam 24 horas por dia e, então, é necessário planejar a circulação dos trens para que eles saiam e cheguem no horário que os pátios ferroviários estejam operando.

O planejamento da circulação de trens em uma ferrovia é tratado na literatura como Problema de Planejamento de Circulação de Trens ou Train Timetabling Problem (TTP) (Cacchiani et al., 2014). Um dos primeiros autores a estudar o TTP foi Szpigel (1972). Dentre os vários trabalhos publicados sobre o TTP destacam-se: Lusby et al. (2011), Harrod (2012) e Cacchiani et al. (2014). Uma revisão mais detalhada sobre os artigos publicados sobre o TTP é apresentada na Seção 2.

Tendo em vista que a Ferrovia Centro Atlântica (FCA) tem uma linha férrea muito extensa, aproximadamente $7.200 \mathrm{~km}$, passando por 316 municípios em sete estados brasileiros (Minas Gerais, Espírito Santo, Rio de Janeiro, Sergipe, Goiás, Bahia, São Paulo) e no Distrito Fede- 
ral, seus gestores decidiram dividi-la em corredores logísticos, que são denominados simplesmente corredor. Cada corredor representa um trecho da ferrovia que está localizado de forma contigua em uma região do Brasil e representa um canal de escoamento de cargas entre certas regiões do Brasil. Assim, a FCA possui os seguintes corredores: Centro-Sudeste, Centro-Leste, Minas-Rio e Minas-Bahia. $\mathrm{O}$ modelo matemático proposto foi testado com dados reais destes quatro corredores.

Foram elaborados 10 cenários de testes, priorizando os trechos de maior movimento de trens por dia, sendo que a elaboração destes cenários será apresentada na Seção 4. O modelo matemático tem por objetivo reduzir o tempo total de viagem de todos os trens. A origem e o destino de cada trem que irá circular na ferrovia são conhecidos e informados para o modelo como parâmetros do mesmo. O modelo foi resolvido usando o solver CPLEX 12.6 com os dados dos 10 cenários e os resultados foram comparados com os dados do executado na FCA. O modelo alcançou redução de tempo de viagem em todos os cenários em relação ao realizado pela FCA.

$\mathrm{O}$ artigo está estruturado em cinco seções, incluindo esta introdução. Na Seção 2 descreve-se o problema de planejamento da circulação de trens e também é realizada uma revisão da literatura sobre o tema. A Seção 3 apresenta o modelo matemático proposto. Na Seção 4 são apresentados os resultados obtidos e as análises. Na Seção 5 são apresentadas as conclusões e por fim as referências.

\section{PROBLEMA DE PLANEJAMENTO DE CIRCULAÇÃO DE TRENS}

O Problema de Planejamento de Circulação de Trens (Train Timetabling Problem (TTP)), diz respeito ao agendamento de trens ou o planejamento da circulação de trens em uma ferrovia (Cacchiani et al., 2014).

A rota de um trem é o conjunto ordenado de segmentos que o trem deve percorrer entre a estação de partida e a estação de destino da sua viagem. Uma linha singela é uma linha férrea que permite a passagem de um único trem a cada momento e pode ser utilizada para as viagens de trens nos dois sentidos. Tendo em vista que a circulação de trens em linhas singelas ocorre em uma única linha para trens em direções opostas, faz-se necessário a construção de um pátio denominado de pátio de cruzamento para permitir que trens em sentidos opostos possam cruzar ao longo da ferrovia. Os pátios de cruzamento também podem ser utilizados para permitir a ultrapassagem de um trem sobre outro. Cada Pátio de Cruzamento possui uma linha paralela à linha de circulação que serve para que um trem possa ser estacionado enquanto o outro trem cruza por ele na linha de circulação.
A linha que compõe o Pátio de Cruzamento deverá ter no mínimo o comprimento do maior trem previsto para circular na ferrovia, incluindo todos os vagões e todas as locomotivas que compõem este trem (Hay, 1982).

Na situação de dois trens se encontrarem em sentidos opostos na mesma linha, um dos trens é desviado para o Pátio de Cruzamento, que no exemplo da Figura 1, é o Trem 02 que aguarda a passagem do Trem 01. Após a passagem do Trem 01, o Trem 02 sai do Pátio de Cruzamento e prossegue viagem.

Vale destacar que, numa linha singela, enquanto um trem ocupa um segmento, usualmente, nenhum outro trem pode ocupá-lo, a não ser em casos excepcionais para trens circulando no mesmo sentido e, estes casos deverão estar descritos nos Regulamentos de Operação Ferroviária da ferrovia. Um conflito é caracterizado quando dois ou mais trens estão prestes a ocupar o mesmo segmento no mesmo tempo. No momento do conflito, o planejamento da circulação de trens deve definir qual deles deve parar para dar passagem ao outro.

Um dos primeiros trabalhos publicados aplicado ao planejamento da circulação de trens é o de Szpigel (1972) que propôs uma abordagem para o planejamento da circulação de trens por meio do problema Job Shop Scheduling solucionado com um algoritmo branch-and-bound. Sauder e Westerman (1983) utilizaram métodos baseados em enumeração numa árvore e cada nível dela representa a solução de um conflito que identificava o trem que devia esperar e desviar. O objetivo do estudo era minimizar o atraso total dos trens. Jovanovic e Harker (1991) propuseram uma estratégia semelhante com experimentos computacionais chegando a instâncias de 100 trens.

Kraay et al. (1991) procuraram determinar o perfil de velocidade de cada trem, minimizando critérios como consumo de combustível, atraso, etc. Para isso o tempo de viagem foi considerado uma variável e buscou-se minimizar os custos de combustível, obviamente dependente da velocidade do trem. Eles apresentaram um modelo de Programação Não Linear Inteira Mista e um método branch-andbound com instâncias com treze estações e 22 trens. Carey (1994) propôs um modelo que permitisse que o modelo de Jovanovic e Harker (1991) viesse a ser utilizado também em casos em que existem múltiplas linhas entre as estações com múltiplas plataformas.

Cai e Goh (1994) estudaram uma linha singela e propuseram uma heurística gulosa e tinham como pressuposto o fato de que todos os trens viajavam na mesma direção, mas não na mesma velocidade e tendo o mesmo destino final.

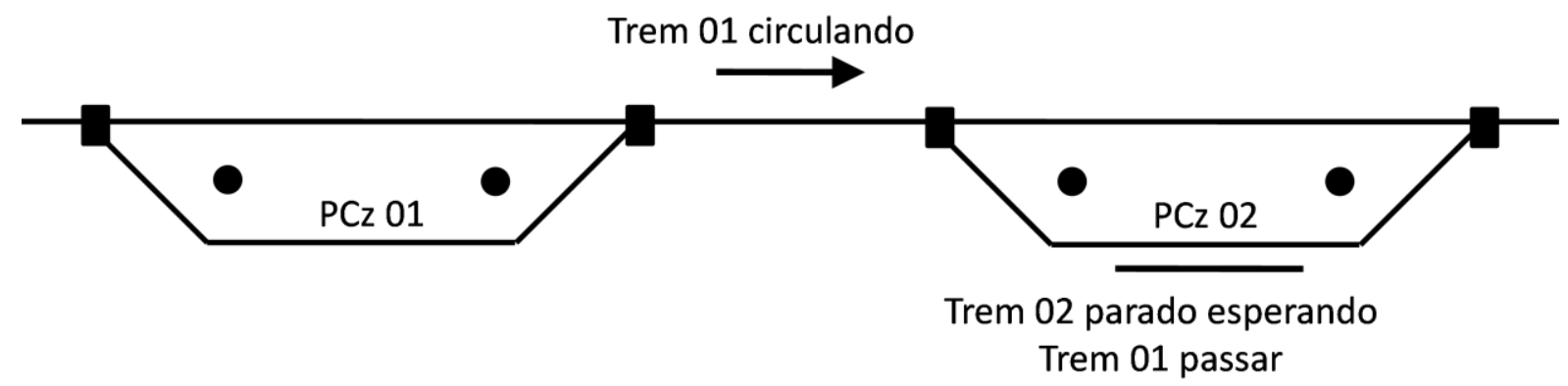

Figura 1. Circulação de trens em linha singela com pátio de cruzamento 
O modelo também podia ser generalizado para atender a possibilidade de ultrapassagem quando os trens têm velocidades diferentes. A heurística considerava os trens em ordem cronológica e assumia que o tempo inicial de partida do pátio de cada trem era conhecido. O estudo apresentou dois exemplos e ambos utilizavam 12 desvios e 12 e 20 trens respectivamente. Carey e Lockwood (1995) basearam-se em Szpigel (1973), entretanto, os trechos entre duas estações eram designados para tráfego em apenas uma direção.

Eles propuseram uma formulação matemática baseada em Programação Linear Inteira Mista (PLIM). Higgins et al. (1996) consideraram um trecho de linha singela bidirecional e apresentaram uma formulação matemática similar a de Carey e Lockwood (1995). Os autores adicionaram uma flexibilidade onde cada trem pode atingir uma velocidade mínima e máxima em cada trecho. O objetivo é minimizar o tempo total de atraso ponderado pela prioridade dos trens. Utilizaram branch-and-bound para resolver instâncias de 30 trens e 12 estações. Higgins et al. (1997) desenvolveram e compararam várias técnicas heurísticas, elevando as instâncias do estudo de 1996 para 50 trens.

Cordeau et al. (1998) forneceram uma visão geral sobre os modelos de roteamento e otimização da programação de trens produzidas até então, classificando-os de acordo com suas características principais. Cai et al. (1998) estenderam o trabalho de Cai e Goh (1994) relaxando o requerimento de que a localização inicial do trem devia ser um pátio e aplicaram o modelo a uma ferrovia asiática com 400 trens/dia e 60 trens simultâneos. Brännlund et al. (1998) propuseram uma formulação packing integer programming e foi utilizada uma rede espaço-tempo com seis diferentes tipos de arco para representar o modelo. O objetivo era minimizar o tempo de espera nos pátios. Foi utilizada Relaxação Lagrangiana com instâncias de 30 trens.

Sahin (1999) estudou o planejamento em tempo real de uma ferrovia Turca com 20 trens e apresentou uma heurística que avalia e resolve os conflitos na ordem em que eles aparecem. Oliveira e Smith (2000) propuseram uma formulação similar à de Szpizel (1973) com o objetivo de minimizar o atraso total e foram incorporadas restrições práticas como forçar dois trens a pararem no mesmo pátio num certo tempo e que um mesmo trem realize múltiplos itinerários. Caprara et at. (2002) estudaram uma ferrovia italiana e modelaram o problema usando multigrafo acíclico direcionado para instâncias com 73 estações e 500 trens com intervalos de 1 minuto. Leal et al. (2004) aplicaram a uma grande ferrovia brasileira os modelos e heurísticas propostos por Szpigel (1973) e por Higgins (1996). Borndörfer et al. (2005) apresentaram formulação similar à Caprara et al. (2002) diferenciando por não considerar corredores interligando as estações e propuseram uma abordagem baseada em leilão para alocar de forma otimizada a capacidade da ferrovia. Caprara et al. (2006) estenderam sua formulação de 2002 para incluir restrições práticas como a capacidade das estações e programação fixa para alguns trens. Resolveram instâncias com 49 estações e 221 trens.

Dall'Orto et al. (2006) apresentaram uma formulação estocástica que busca otimizar o problema de serviços de despachos num terminal e propuseram uma solução com programação dinâmica e duas meta-heuristicas Tabu Search com um mecanismo de aprendizagem. Borndörfer e
Schlechte (2007b) reconheceram que os modelos anteriores acrescentavam muitas restrições a fim de resolver conflitos, como Borndörfer et al. (2005) que tem relaxações fracas de programação linear. Eles adicionam mais variáveis ao invés de restrições e definiram variáveis binárias para definir a seleção do trajeto dos trens e para ditar a configuração dos arcos em cada trecho. Instâncias de até 570 trens num trecho de uma ferrovia alemã foram resolvidas.

Tornquist (2006) forneceu uma visão geral sobre planejamento da circulação de trens e são revistos 48 abordagens publicadas entre 1973 e 2005 , classificando-as quanto ao tipo de problema, o mecanismo de solução e o tipo de avaliação. Num estudo comparativo Borndorfer e Schlechte (2007a) basearam a configuração da via na formulação dada por Borndorfer e Schlechte (2007b) e detalharam as formulações de empacotamento. As formulações de empacotamento são aquelas que não incluem variáveis que configuram arcos e tipicamente preveem conflitos de trens através da inclusão de constante de empacotamento. Experimentos computacionais foram baseados em uma ferrovia alemã que consistia em 37 estações com 120 trechos. O objetivo dos problemas era agendar o máximo de trens possível.

Tornquist e Persson (2007) consideraram o reagendamento de trens numa ferrovia Sueca quando ocorrem eventos que mudem o planejamento na circulação. Eles utilizaram uma formulação matemática baseada em PLIM e o aplicaram a uma ferrovia sueca com 169 estações e 508 trens. Cacchiani et al. (2008) utilizaram uma rede espaçotempo similar à da proposta por Caprara et al. $(2002,2006)$ e empregaram um algoritmo branch-and-cut-and-price bem como duas aproximações heurísticas resolvendo instâncias de 11 casos reais de uma ferrovia italiana, com 102 estações e 221 trens.

Cacchiani et al. (2010) generalizaram as abordagens anteriores de Cacchiani et al. (2008) e Caprara et al. (2002, 2006) para o caso de uma rede ferroviária geral, onde já existe a programação fixa para trens de passageiros e o objetivo é introduzir o maior número de trens de carga possível. Os autores apresentaram uma formulação de Programação Linear Inteira e propuseram uma heurística Lagrangiana. Resultados em instâncias reais foram apresentados.

Liu e Kozan (2011) apresentaram um algoritmo para solução de problemas de agendamento de trens prioritários e não prioritários numa mesma rede ferroviária. Experimentos computacionais extensos demonstram que o método proposto é promissor. Lusby et al. (2011) apresentaram uma revisão sobre o planejamento da circulação de trem.

Tornquist (2012) complementou a abordagem de Tornquist e Persson (2007) e apresentou um algoritmo guloso para problemas de reagendamento de trens. Harrod (2012) apresentou em seu artigo uma revisão bibliográfica sobre planejamento de trens. Cacchiani e Toth (2012) apresentaram um levantamento sobre os principais estudos que lidam com TTP, mostrando as diferenças entre os modelos e destacaram as soluções que evitam a propagação de atrasos.

Harrod (2013) apresentou um método de otimização global de despacho de trens, considerando um trem prioritário de alta velocidade numa rede de linha singela com vários trens mais lentos. Reimann e Leal (2013) propuseram uma meta-heurística Colônia de Formigas para o problema de programação da circulação de trem. 
Fabris et al. (2014) apresentaram uma aplicação em larga escala de uma heurística, considerando um modelo mesoscópico da infraestrutura da rede ferroviária do norte da Itália, permitindo assim uma precisão maior nas análises. Foram realizados testes de capacidade máxima de transporte de cargas combinado com transporte de passageiros. Cacchiani et al. (2014) deram uma visão geral dos dois sistemas fundamentais de otimização de um sistema ferroviário: O TTP que é o agendamento de trens ou o planejamento da circulação de trens e o TPP (Train Platforming Problem) que é a programação de trens nas estações.

\section{MODELO MATEMÁTICO PROPOSTO}

O modelo matemático proposto foi elaborado com base no problema Job Shop (Pinedo, 2009). Para o modelo matemático, foi considerado que a ferrovia é dividida em segmentos, Figura 2. Assim, foram definidos dois tipos de segmentos. O primeiro tipo de segmento é o que representa a linha singela propriamente dita, que no caso da Figura 2 são os trechos 1, 4 e 7. O segundo tipo de segmento é o que representa o pátio de cruzamento e, então, cada pátio de cruzamento sempre terá dois segmentos. No caso da Figura 2, o pátio de cruzamento PC01 é representado pelos segmentos 2 e 3 e o pátio de cruzamento PC02 é representado pelos segmentos 5 e 6 .

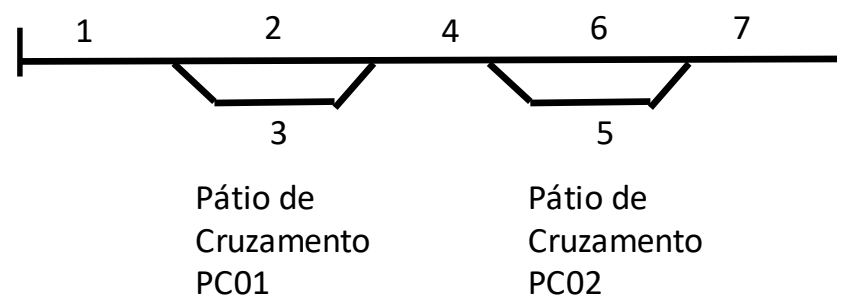

Figura 2. Definição de segmentos sob a ótica do modelo matemático proposto

Analogamente ao modelo Job Shop, as tarefas são as viagens dos trens, as máquinas são os segmentos a serem percorridos pelo trem e o tempo de processamento é o tempo médio de viagem do trem em cada segmento. Podese avaliar a qualidade do planejamento da circulação de trens por meio do makespan, que no caso da ferrovia representa a soma do tempo de término de viagem de todos os trens. Como no modelo Job Shop, uma tarefa, que é o trem, não pode ocupar uma máquina no mesmo tempo, que é o segmento, e, assim, garante-se que somente um trem poderá ocupar um segmento e que um trem deverá aguardar em um segmento, uma linha do pátio de cruzamento, até que seja liberado o segmento que ele pretende viajar. Apresenta-se a seguir a formulação matemática em cinco partes: conjuntos, parâmetros, variáveis de decisão, função objetivo e restrições.

Conjuntos:

$N$ Conjunto de todos os trens que circularão na ferrovia;

$S$ Conjunto de todos os segmentos da ferrovia;

$S_{i}$ Conjunto dos segmentos percorridos pelo trem $i \in N$ para realizar sua viagem, $S_{i} \subseteq S$; e
$S \mathrm{~A}_{i}$ - Conjunto auxiliar para lógica do modelo que equivale ao conjunto $S_{i}$, porém o número de segmentos é igual ao número de segmentos de $S_{i}$ menos 1.

Parâmetros:

$p_{i, s}$ - Tempo médio de viagem do trem $i \in N$ no segmento $s \in S_{i}$;

$n_{i}$ - Número de segmentos percorridos pelo trem $i \in N$;

$\alpha_{i}$ - Momento limite permitido de adiantamento do horário da partida do trem $i \in N$;

$\beta_{i}$ - Momento limite permitido de atraso do horário da partida do trem $i \in N$;

$\delta_{i}$ - Momento limite permitido de adiantamento do horário de chegada do trem $i \in N$; e

$\phi_{i}$ - Momento limite permitido de atraso do horário da chegada do trem $i \in N$.

Variáveis de decisão:

$y_{i, s}$ - Momento em que o trem $i \in N$ termina de percorrer o segmento $s \in S_{i}$; e

$x_{i, j, s}$ - Define a precedência do trem $i \in N$ em relação ao trem $j \in N$ em um segmento $s \in S$. Variável binária que assume o valor 1 se o trem $i \in N$ precede o trem $j \in N$, no segmento $s \in S_{i}$ e 0 , caso contrário.

Antes de apresentar a função objetivo e as restrições do problema, são explicados por meio de um exemplo, Figura 3, os principais parâmetros e variáveis de decisão do modelo proposto. Tem-se uma ferrovia com 19 segmentos, $S=\{1, \ldots, 19\}$. O trem (trem 1) viaja na ferrovia no sentido do segmento 13 para o segmento 4 , portanto sete segmentos, $n_{1}=7$. O conjunto de segmentos a serem percorridos pelo trem 1 é $S_{1}=\{13,11,10,9,7,5,4\}$. A partida do trem se dá no primeiro segmento, 13 , e o tempo de término de viagem no primeiro segmento será $y_{1,13}$. A chegada do trem se dá no sétimo segmento, $n_{1}=7$, e, assim o último segmento será 4 e o tempo de término de viagem no último segmento será $y_{1,4}$, que também será o tempo de término de toda a viagem do trem 1. Por fim, pode se ver o parâmetro $p_{1,4}$ que representa o tempo médio de viagem do trem 1 no segmento 4.

Função Objetivo

Minimizar $\sum_{i \in N} y_{i, n_{i}}$ 


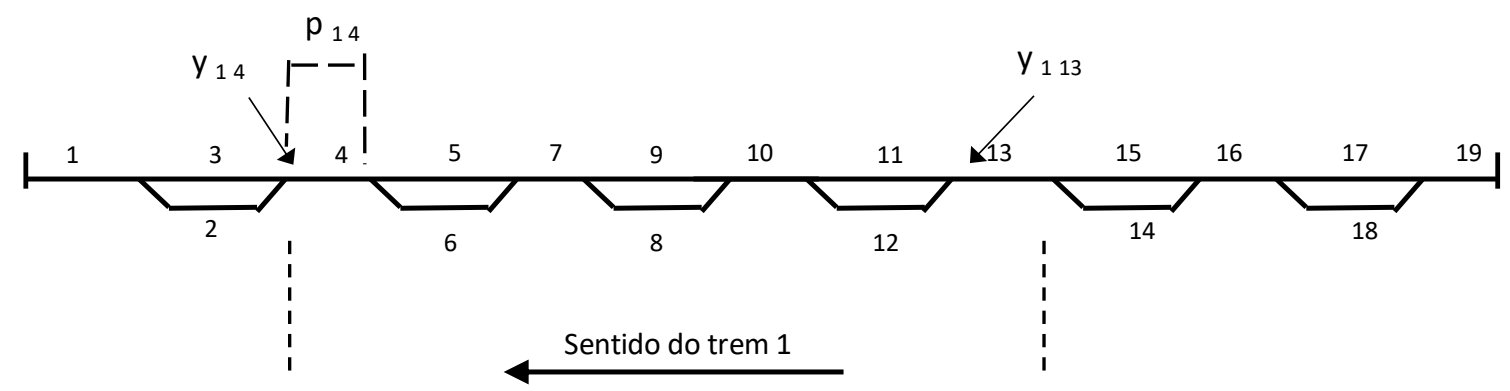

Figura 3. Principais parâmetros e variáveis de decisão

Sujeito a:

$$
\begin{array}{cl}
y_{i,(s+1)} \geq y_{i, s}+p_{i,(s+1)} & \forall i \in N, s \in S \mathrm{~A}_{i} \\
y_{j, s} \geq y_{i, s}+p_{j, s} & \forall i, j \in N, i \neq j, \\
-M\left(1-x_{i, j, s}\right) & s \in S_{i} \\
y_{i, s} \geq y_{j, s}+p_{i, s} & \forall i, j \in N, i \neq j, \\
\quad-M x_{i, j, s} & s \in S_{i} \\
\alpha_{i} \leq\left(y_{i, 1}-p_{i, 1}\right) \leq \beta_{i} & \forall i \in N \\
\delta_{i} \leq y_{i, n_{i}} \leq \phi_{i} & \forall i \in N \\
y_{i, s}>0 & \forall i \in N, s \in S_{i} \\
y_{i, s}=0 & \forall i \in N, s \notin S_{i} \\
x_{i, j, s}=0 & \forall i, j \in N, i=j, \\
x_{i, j, s}=0 & s \in S \\
x_{i, j, s} \in\{0,1\} & \forall i, j \in N, i \neq j, \\
& s \notin\left\{S_{i} \cap S_{j}\right\} \\
& \forall i, j \in N, s \in S
\end{array}
$$

A função objetivo, Equação (1), representa a soma do momento de término do último trecho da viagem, $y_{i, n_{i}}$, de todos os trens. A função objetivo deve ser minimizada.

As Restrições (2) garantem que o momento em que o trem $i \in N$ termina de percorrer o segmento $s \in S A_{i}$ seja maior ou igual ao momento em que o mesmo trem termina de percorrer segmento $s \in S A_{i}$. As Restrições (3) e (4) estabelecem a precedência de um trem em relação ao outro.

As Restrições (5) garantem que cada trem inicie sua viagem dentro do intervalo compreendido entre o momento limite permitido de adiantamento do horário de partida do trem e o momento limite permitido de atraso do horário de partida do trem. As Restrições (6) garantem que cada trem termine sua viagem dentro do intervalo compreendido entre o momento limite permitido de adiantamento do horário de chegada do trem e o momento limite permitido de atraso do horário de chegada do trem.

As Restrições (7) garantem que se um trem $i \in N$ quando percorrendo um seguimento $s \in S_{i}$, o momento no qual o trem termina a viagem em cada sequência de segmentos da sua rota seja sempre maior que zero. As Restrições (8) garantem que caso um segmento não seja percorrida pelo trem, $s \notin S_{i}$, então o tempo de término de viagem neste segmento será igual à zero, isto é, o trem não circula neste segmento. As Restrições (9) garantem que a variável $x_{i, j, s}$ assumirá o valor 0 se $i=j$, ou seja, o mesmo trem e, portanto, não pode haver precedência entre um trem sobre ele mesmo. As Restrições (10) garantem que a variável $x_{i, j, s}$ assumirá o valor 0 se o segmento $s$ não pertencer ao conjunto intercessão dos segmentos do trem $i$ e do trem $j$, $s \notin\left\{S_{i} \cap S_{j}\right\}$. As Restrições (11) garantem que a variável $x_{i, j, s}$ assumirá valores 0 ou 1 .

\section{APRESENTAÇÃO E ANÁLISE DE RESULTADOS}

Os dados utilizados para os testes foram fornecidos pela ferrovia FCA e são referentes aos meses de janeiro a junho de 2014. Os dados foram coletados via acesso ao banco de dados do sistema UNILOG que é utilizado para o planejamento e registro das informações operacionais da ferrovia. Foram desenvolvidas diversas querys SQL onde foram selecionados os trens que circularam na ferrovia por dia em cada corredor. Após esta extração dos dados, os mesmos foram tratados no Excel visando identificar os dias de maior circulação de trens em cada corredor. Destes dados, foram escolhidos os 10 dias de maior circulação de trens e foram elaborados 10 cenários de testes que são apresentados na Tabela 1 onde a coluna No de Trens representa o total de trens que circularam em um dia na ferrovia nos dois sentidos.

Além disso, foram levantados junto à operação os tempos médios de viagem em cada segmento da ferrovia dos diferentes tipos de trens, i.e., cargueiro de soja, minério, cargueiro de combustível dentre outros. Como cada trem que circula na ferrovia é de um tipo específico, então podese definir a velocidade média de cada trem em cada segmento que ele irá circular na ferrovia.

Utilizou-se o solver CPLEX 12.6 para resolver o modelo matemático proposto usando um computador Intel i7, com $8 \mathrm{~GB}$ de memória RAM. Foi estabelecido um tempo limite de 7.200 segundos (2 horas) para resolução de cada um dos modelos associados aos cenários apresentados na Tabela 1.

Os resultados obtidos foram comparados com os dados reais da circulação de trens da FCA, que neste artigo são definidos como resultados do Caso Real. 
Modelo Matemático para o Planejamento da Circulação de Trens em Ferrovias de Linha Singela

Tabela 1. Cenários elaborados a partir de dados reais da FCA

\begin{tabular}{|c|c|c|c|c|c|c|}
\hline Cenário & Nome do Corredor & Trecho Inicial & Trecho Final & $\begin{array}{c}\text { Extensão } \\
\text { total }(\mathbf{k m})\end{array}$ & $\mathbf{N}^{0}$ Pátios Cruzamento & $\mathbf{N}^{o}$ de Trens \\
\hline 1 & Minas Rio & $\operatorname{Arcos}$ & Barra Mansa & 467 & 29 & 13 \\
\hline 2 & Centro Sudeste & Ribeirão Preto & Paulínia & 274 & 16 & 19 \\
\hline 3 & Minas Bahia & Pirapora & Wilson Lobato & 350 & 17 & 6 \\
\hline 4 & Centro Leste & Brejo Alegre & Eldorado & 733 & 61 & 16 \\
\hline 5 & Centro Leste & Roncador Novo & Brejo Alegre & 174 & 11 & 4 \\
\hline 6 & Centro Sudeste & Uberlândia & Ribeirão Preto & 316 & 16 & 11 \\
\hline 7 & Minas Rio & B. de Camargos & Três Rios & 175 & 15 & 11 \\
\hline 8 & Minas Bahia & Pirapora & Wilson Lobato & 350 & 17 & 8 \\
\hline 9 & Centro Leste & Garça de Minas & Eldorado & 285 & 30 & 12 \\
\hline 10 & Centro Sudeste & Ribeirão Preto & Paulínia & 274 & 16 & 17 \\
\hline
\end{tabular}

Na Tabela 2 são apresentados os resultados alcançados pelo modelo proposto utilizando o CPLEX 12.6 e o apurado no Caso Real. A coluna "Tempo de Execução" apresenta o tempo computacional utilizado pelo CPLEX. A coluna "Tempo de Viagem" exibe o tempo total de viagem de todos os trens. A coluna "Tempo" apresenta a redução de tempo de viagem alcançada pelo modelo matemático em relação ao realizado no Caso Real. A redução alcançada em termos percentuais é apresentada na coluna "Percentual". O Tempo total de viagem do trem engloba a soma de todo o tempo de circulação em cada trecho mais a soma do tempo parado nos pátios de cruzamento.

Nos resultados apresentados na Tabela 2 para o Cenário 1 , observou-se uma redução de $23,84 \%$. O CPLEX encontrou a uma solução ótima num tempo de execução de 11,2 segundos. Tendo em vista que para alguns trens os ganhos foram muito expressivos em relação ao alcançado no Caso Real, optou-se por uma análise detalhada da operação destes trens. Após a análise, detectou-se que na circulação destes trens, eles ficaram parados ao longo do trecho por diversos motivos que não tinham relação com o cruzamento de trens.

É interessante notar que o CPLEX conseguiu resolver o maior cenário em termos de pátio de cruzamento (Cenário 4) com 16 trens e 61 pátios de cruzamento, em 52 minutos. Todos os outros cenários foram resolvidos num tempo inferior a 25 minutos. Esses são tempos factíveis para o uso do modelo proposto pela FCA no planejamento diário. Destaca-se ainda que o apesar do Cenário 2 ter menos trens que o Cenário 10, ambos pertencentes ao mesmo corredor, o tempo de processamento do CPLEX para o Cenário 2 foi maior que o Cenário 10. Isto pode ter ocorrido provavelmente por conta dos trens de cada cenário serem diferentes, pois são trens de dias diferentes, e assim, possuírem horas de partida, origens e destinos diferentes e, por conta disto, o grau de dificuldade do modelo convergir para uma solução ótima pode ser diferente, não sendo possível comparar os tempos de processamento do CPLEX entre dois cenários.

Tabela 2. Comparação dos Resultados do Modelo Proposto x Caso Real

\begin{tabular}{|c|c|c|c|c|c|}
\hline \multirow{3}{*}{ Cenário } & \multicolumn{2}{|c|}{$\begin{array}{c}\text { CPLEX } \\
\text { Modelo Proposto }\end{array}$} & \multirow{3}{*}{$\begin{array}{c}\text { Caso Real } \\
\text { Tempo de Viagem } \\
\text { (min) }\end{array}$} & \multicolumn{2}{|c|}{ Redução Tempo de Viagem (Modelo x Caso Real) } \\
\hline & \multirow{2}{*}{$\begin{array}{l}\text { Tempo de } \\
\text { Execução } \\
\text { (s) }\end{array}$} & \multirow{2}{*}{$\begin{array}{c}\text { Tempo de } \\
\text { Viagem } \\
(\min )\end{array}$} & & & \\
\hline & & & & Tempo (min) & Percentual (\%) \\
\hline 1 & 11,2 & $7.123,0$ & $9.353,0$ & $2.230,0$ & 23,8 \\
\hline 2 & 183,3 & $11.915,0$ & $25.536,0$ & $13.621,0$ & 53,3 \\
\hline 3 & 2,7 & $4.460,0$ & $6.566,0$ & $2.106,0$ & 32,1 \\
\hline 4 & $3.162,2$ & $26.725,0$ & $36.928,0$ & $10.203,0$ & 27,6 \\
\hline 5 & 1,4 & $1.286,0$ & $2.941,0$ & $1.655,0$ & 56,3 \\
\hline 6 & 7,4 & $6.289,0$ & $13.333,0$ & $7.044,0$ & 52,8 \\
\hline 7 & 9,4 & $5.719,0$ & $6.295,0$ & 576,0 & 9,2 \\
\hline 8 & 4,7 & $6.318,0$ & $8.481,0$ & $2.163,0$ & 25,5 \\
\hline 9 & 5,9 & $5.551,0$ & $6.690,0$ & $1.139,0$ & 17,0 \\
\hline 10 & $1.467,8$ & $10.974,0$ & $26.293,0$ & $15.319,0$ & 58,3 \\
\hline
\end{tabular}


PEREIRA, E.P.; ROSA, R.A.; BERGER, P.P.; CARVALHO, J.T.; RIBEIRO, G.M.

Tabela 3. Comparação Resultados do Modelo Proposto x Caso Real excluindo as Horas Paradas

\begin{tabular}{|c|c|c|c|c|c|c|}
\hline \multirow[b]{2}{*}{ Cenário } & \multicolumn{2}{|c|}{$\begin{array}{c}\text { CPLEX } \\
\text { Modelo Proposto }\end{array}$} & \multicolumn{2}{|c|}{ Caso Real } & \multicolumn{2}{|c|}{$\begin{array}{l}\text { Redução Tempo de Viagem } \\
\text { (Modelo x Caso Real) }\end{array}$} \\
\hline & $\begin{array}{l}\text { Tempo de } \\
\text { Execução }\end{array}$ & $\begin{array}{l}\text { Tempo de } \\
\text { Viagem } \\
\text { (min) }\end{array}$ & $\begin{array}{l}\text { Horas Paradas } \\
\qquad(\min )\end{array}$ & $\begin{array}{c}\text { Tempo de Viagem } \\
\text { sem Hora Parada } \\
\text { (min) }\end{array}$ & Tempo (min) & Percentual (\%) \\
\hline 1 & 11,2 & $7.123,0$ & $1.429,0$ & $7.924,0$ & 801,0 & 10,1 \\
\hline 2 & 183,3 & $11.915,0$ & $11.709,0$ & $13.827,0$ & $1.912,0$ & 13,8 \\
\hline 3 & 2,7 & $4.460,0$ & 797,0 & $5.769,0$ & $1.309,0$ & 22,7 \\
\hline 4 & $3.162,2$ & $26.725,0$ & $7.950,0$ & $28.978,0$ & $2.253,0$ & 7,8 \\
\hline 5 & 1,4 & $1.286,0$ & $1.567,0$ & $1.374,0$ & 88,0 & 6,4 \\
\hline 6 & 7,4 & $6.289,0$ & $5.180,0$ & $8.153,0$ & $1.864,0$ & 22,9 \\
\hline 7 & 9,4 & $5.719,0$ & 365,0 & $5.930,0$ & 211,0 & 3,6 \\
\hline 8 & 4,7 & 6318 & 751 & $7.730,0$ & $1.412,0$ & 18,3 \\
\hline 9 & 5,9 & 5551 & 819 & $5.871,0$ & 320,0 & 5,5 \\
\hline 10 & $1.467,8$ & 10974 & 12849 & $13.444,0$ & $2.470,0$ & 18,4 \\
\hline
\end{tabular}

Assim, foi realizada uma análise do indicador Trem Hora Parado (THP) utilizado pela FCA para controlar os motivos que ocasionaram as paradas dos trens. O THP é um indicador utilizado por grande parte das ferrovias para controlar os motivos que ocasionaram as paradas dos trens e o tempo gasto em cada uma delas. Foram consideradas paradas dos tipos: defeito na locomotiva, quebra de trilho, etc. O tempo gasto em cada parada foi denominado neste artigo como Horas Paradas. Do tempo total registrado pela ferrovia para cada trem foi subtraído o tempo de Horas Paradas gerando um tempo total efetivo de circulação do trem. Isso foi realizado para que fosse possível fazer a comparação entre os resultados obtidos pelo CPLEX e pela ferrovia.
Isso se deve ao fato que essas Horas Paradas representam problemas que ocorreram durante a condução do trem e não no planejamento da ferrovia.

Na Tabela 3 pode-se observar que em todos os cenários o CPLEX conseguiu ganhos em relação ao Caso Real variando de $3,6 \%$ a $22,9 \%$. No gráfico da Figura 4 são apresentados os tempos de viagem registrados no Caso Real e o tempo obtido pelo CPLEX para cada cenário. Ainda no mesmo gráfico, acima das barras que representam o tempo de viagem, são apresentadas as reduções em percentual do tempo de viagem alcançadas em relação ao Caso Real.

É interessante notar que o CPLEX conseguiu resolver o maior cenário em termos de pátio de cruzamento (Cenário 4) com 16 trens e 61 pátios de cruzamento, em 52 minutos.

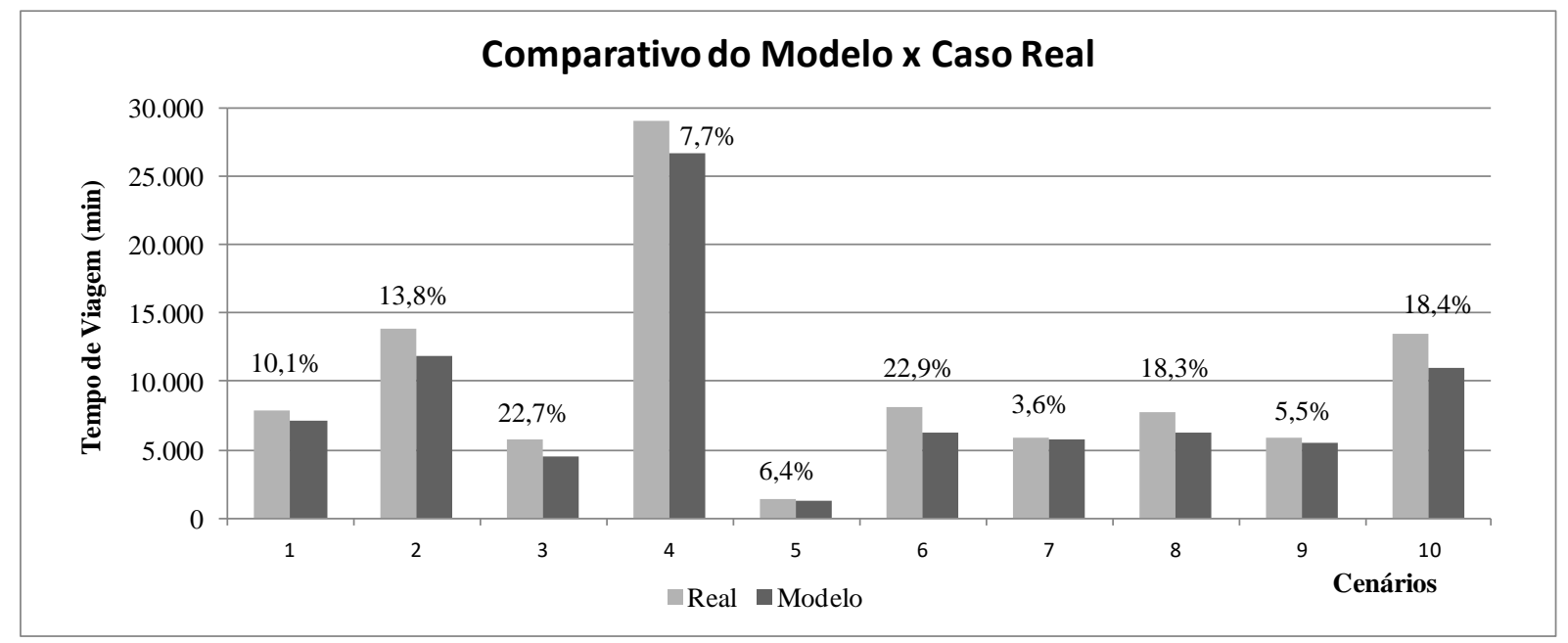

Figura 4. Comparação Modelo $\times$ Caso Real 
Todos os outros cenários foram resolvidos num tempo inferior a 25 minutos. Esses são tempos factíveis para o uso pela FCA do modelo proposto no planejamento diário da circulação de trens.

Tendo em vista que todos os testes foram realizados com dados reais da ferrovia estudada e que a mesma não possui registros dos trens que não circularam, não há a possibilidade de se analisar o aumento de trens circulando na ferrovia, ou seja, o aumento efetivo de capacidade em número de trens. No entanto, as reduções nos tempos de viagem apresentadas na Tabela 3 e na Figura 4 mostraram que os trens concluíram suas viagens de forma mais rápida, em um tempo menor, e, consequentemente, pode-se supor que a ferrovia tendo maior disponibilidade de linha não ocupada permitiria um possível aumento de trens circulando utilizando estes momentos onde a linha ficou sem ocupação.

A redução total do tempo de viagem foi calculada somando-se toda a coluna Redução Tempo de Viagem (Modelo x Caso Real) - Tempo e chegou-se, então, a uma redução de 12.640,0 minutos para os 10 cenários. Tomando como base que o consumo médio de combustível de uma locomotiva típica da FCA parada (ponto morto) é de 15 litros/hora $(0,25$ litros/minuto) e, considerando que a locomotiva não é desligada enquanto estacionada em um pátio de cruzamento, pode-se inferir que, caso o modelo proposto tivesse sido utilizado para o planejamento dos 10 cenários analisados, por dia seriam economizados 3.098,9 litros de combustível (sem considerar o combustível para os procedimentos de parada e para a partida dos trens). Ampliandose estes valores para um ano, pode-se economizar pouco mais de 1,1 milhões de litros de combustível. Considerando ainda que o preço médio atual de um litro de óleo diesel para grandes empresas é de $\mathrm{R} \$ 2,23$, obtém-se uma economia da ordem de R $\$ 2,5$ milhões por ano.

Por dados estatísticos da FCA, sabe-se que são emitidos em média $2,7 \mathrm{Kg}$ de $\mathrm{CO}_{2}$ equivalente por litro de diesel consumido pelas locomotivas da FCA. Assim, utilizando os valores de redução de combustível encontrados, pode-se estimar uma redução de emissão na atmosfera de aproximadamente 3,0 milhões de quilos de $\mathrm{CO}_{2}$ equivalente.

\section{CONCLUSÃO}

Este artigo apresentou um modelo matemático que tem por objetivo a redução do tempo total de viagem de todos os trens e, indiretamente, o modelo leva a uma redução do tempo parado em pátios de cruzamento de todos os trens. O modelo matemático proposto neste artigo realiza o planejamento da circulação de trens em uma ferrovia de linha singela e seus resultados, quando comparados com casos reais executados pela ferrovia FCA, apresentaram ganhos de 3,6 a 22,9\%. Como visto, esses resultados podem gerar ganhos financeiros da ordem de $\mathrm{R} \$ 2,5$ milhões por ano somente nos cenários testados e ainda uma redução de emissão de $\mathrm{CO}_{2}$ da ordem de 3,0 milhões de quilos de $\mathrm{CO}_{2}$ equivalente.

Apesar deste artigo não analisar as questões de prioridade dos trens, pois esta situação não ocorre atualmente na FCA, o modelo matemático permite que sejam implementadas prioridades bastando para isso estabelecer que os limites de horário de antecipado e de horário atrasado do horário de partida sejam iguais à hora desejada da partida do trem e que os limites de adiantamento e de atraso do horário de chegada sejam iguais à hora desejada de chegada do trem. Esta situação é importante, pois a FCA planeja em futuro breve retornar com os trens de passageiros e com os trens denominados expressos, trens estes que tem horário para partir e para chegar ao destino.

É importante destacar ainda, que o modelo matemático proposto pode ser aplicado a diversas ferrovias do Brasil e do mundo que possuam linha singela. Pelos resultados alcançados, sabe-se que o CPLEX consegue resolver diversos cenários de maneira ótima que são compatíveis com a realidade da maioria das ferrovias do Brasil. No entanto, para resolver cenários de maior escala, como por exemplo, ferrovias com maior circulação de trens e/ou com uma maior quantidade de pátios de cruzamento, sugerem-se como trabalhos futuros o desenvolvimento de meta-heurísticas para que soluções possam ser obtidas em um tempo computacional aceitável. Sugere-se ainda que sejam consideradas as incertezas inerentes ao transporte ferroviário e, para tanto, sugere-se que os modelos matemáticos incorporem estas incertezas e modelos estocásticos e/ou modelos robustos para o problema.

\section{AGRADECIMENTOS}

Os autores agradecem à FAPES (processo 75528452/2016) e ao CNPq (477148/2011-5 e 313408/2014-9) pelo apoio financeiro e à empresa VLI S/A e à ferrovia FCA pelo fornecimento dos dados para avaliação do modelo.

\section{REFERÊNCIAS}

BORNDÖRFER, R.; Grötschel, M.; Lukac, S.; Mitusch, M.; Schlechte, T.; Schultz, S.; Tanner, A. An auctioning approach to railway slot allocation. Technical Report 05-45, Konrad-ZuseZentrumfür Information stechnik Berlin, 2005.

BORNDÖRFER, R.; Schlechte, T. Models for railway track allocation. Technical Report 07-02, Konrad-Zuse-Zentrumfür Informations technik Berlin, 2007a.

BORNDÖRFER, R.; Schlechte, T. Solving railway track allocation problems. Technical Report 07-20, Konrad-ZuseZentrumfür Information stechnik Berlin, 2007b.

BRÄNNLUND, U.; Lindberg, P. O.; Nõu, A.; Nilsson, J. E. Railway timetabling using lagrangian relaxation. Transportation Science 32(4):358-369, 1998. DOI: 10.1287/trsc.32.4.358

CACCHIANI, V.; Caprara, A.; Toth, P. A column generation approach to train timetabling on a corridor. 4OR 6:125-142, 2008. DOI: $10.1007 / \mathrm{s} 10288-007-0037-5$

CACCHIANI, V.; Caprara, A.; Toth, P. Scheduling extra freight trains on railway networks. Transportation Research Part B 44(2):215-231, 2010. DOI: 10.1016/j.trb.2009.07.007

CACCHIANI, V.; Toth, P. Nominal and robust train timetabling problems. European Journal of Operational Research, Vol. 219(3):727-737, 2012. DOI: 10.1016/j.ejor.2011.11.003

CACCHIANI, V.; Galli, L.; Toth, P. A tutorial on non-periodic train timetabling and platforming problems. EURO Journal on Transportation and Logistics. Springer-Verlag Berlin Heidelberg, 2014. DOI: 10.1007/s13676-014-0046-4 
CAI, X..; Goh, C. J. A fast heuristic for the train scheduling problem. Computers \& Operations Research 21 (5): 499-510, 1994.

CAI, X.; Goh, C. J.; Mees, A. I. Greedy heuristics for rapid scheduling of trains on a single track. IIE Transactions 30:481493, 1998.

CAPRARA, A.; Fischetti, M.; Toth, P.; Modeling and solving the train timetabling problem. Operations Research 50(5):851861, 2002.

CAPRARA, A.; Monaci, M.; Toth, P.; Guida, P.L. A Lagrangian heuristic alogorithm for a real-world train timetabling problem. Discrete Applied Mathematics 154:738-753, 2006. doi:10.1016/j.dam.2005.05.026

CAREY, M. A model and strategy for train pathing with choice of lines, platforms, and routes. Transportation Research Part B 28(5):333-353, 1994.

CAREY, M.; Lockwood, D. A model, algorithms and strategy for train pathing. The Journal of the Operational Research Society 46(8):988-1005, 1995.

CNT (CONFEDERAÇÃO NACIONAL DO TRANSPORTE). Pesquisa CNT de Ferrovias 2011. Brasília: CNT, 2011.

CNT (CONFEDERAÇÃO NACIONAL DO TRANSPORTE). Transporte e Economia - O Sistema Ferroviário Brasileiro. Brasília: CNT, 2013.

CORDEAU, J. F. ; Toth, P. ; Vigo, D. A survey of optimization models for train routing and scheduling. Transportation Science Vol. 32(4):380-404, 1998. doi.org/10.1287/trsc.32.4.380

DALL'ORTO, L. C.; Crainic, T. G.; Leal, J. E.; Powell, W. B. The single-node dynamic service scheduling and dispatching problem. European Journal of Operational Research, vol. 170(1), p. 1-23, 2006. DOI: 10.1016/j.ejor.2004.06.016

FABRIS, S.; Longo, G. ; Medeossi, G. ; Pesenti, R. Automatic generation of railway timetables based on a mesoscopic infrastructure model. Journal of Rail Transport Planning and Management 4:2-13, 2014. DOI: 10.1016/j.jrtpm.2014.04.001

GEIPOT (Empresa Brasileira de Planejamento de Transportes) Anuário Estatístico dos Transportes, 2001.

HARROD, S. S. A tutorial on fundamental model structures for railway timetable optimization. Surveys in Operations Research and Management Science, vol. 17 p. 85-96, 2012. doi:10.1016/j.sorms.2012.08.002

HARROD, S. S. A Method for Robust Strategic Railway Dispatch Applied to a Single Track Line. Transportation Journal, vol. 52(1) p. 26-50, 2013. DOI: 10.1353/tnp.2013.0002

HAY, W. Railroad Engineering. 2nd ed. New York: WileyInterscience, 1982.

HIGGINS, A.; Kozan, E.; Ferreira, L. Optimal scheduling of trains on a single line track. Transportation Research Part B 30(2):147-161, 1996.

HIGGINS, A.; Kozan, E.; Ferreira, L. Heuristic techniques for single line train scheduling. Journal of Heuristics 3:43-62, 1997.
JOVANOVI'C, D.; Harker, P. T. Tactical scheduling of rail operations: the scan I system. Transportation Science 25(1):4664, 1991.

KRAAY, D.; Harker, P. T.; Chen, B. Optimal pacing of trains in freight railroads: model formulation and solution. Operations Research 39 (1):82-99, 1991.

LEAL, J. E.; Soares, A. C.; Nunes, L. S. N. Uma abordagem heurística para o problema de programação de trens em linhas singelas. In: XVIII ANPET - Congresso de Pesquisa e Ensino em Transportes - Florianópolis,2004.

LIU, S. Q.; Kozan, E. Scheduling Trains with Priorities: A NoWait Blocking Parallel-Machine Job-Shop Scheduling Model. Transportation Science Vol. 45(2):175-198, 2011. DOI: $10.1287 /$ trsc. 1100.0332

LUSBY, R. M.; Larsen, J. Ehrgott, M.; Ryan, D. Railway track allocation: models and methods. OR Spectrum; vol. 33, p. 843883, 2011. DOI: 10.1007/s00291-009-0189-0

OLIVEIRA, E.; Smith, B. M. A Job shop scheduling model for the single track-railway timetabling problem. Technical Report 21, University of Leeds, 2000.

PINEDO, M. L. Planning and Scheduling in Manufacturing and Services. $2^{\text {nd }}$ ed. Springer Series Operation Research: New York, USA, 2009.

REIMANN, M., Leal, J. E. Single line train scheduling with ACO. In Proceeding EvoCOP'13 Proceedings of the 13th European conference on Evolutionary Computation in Combinatorial Optimization. p. 226-237 Springer-Verlag Berlin, Heidelberg 2013. DOI: 10.1007/978-3-642-37198-1

SAHIN, I. Railway traffic control and train scheduling based on inter-train conflict management. Transportation Research Part B 33:511-534, 1999.

SAUDER, R.; Westerman, W. M. Computer aided train dispatching: decision support through optimization. Interfaces 6:24-37, 1983. DOI: 10.1287/inte.13.6.24

SZPIGEl, B. Optimal train scheduling on a single line railway. Operation Research: 72:344-351.1973.

SZPIGEL, B. Sequenciamento de Trens. Dissertação de Mestrado. Pontifícia Universidade Católica do Rio de Janeiro: Rio de Janeiro, 1972.

TÖRNQUIST, J., 2006. Computer-based decision support for railway traffic scheduling and dispatching: a review of models and algorithms. 5th Workshop on Algorithmic Methods and Models for Optimization of Railways, (Dagstuhl, Germany) Dagstuhl Research Online Publication Server, 2006. DOI: 10.4230/OASIcs.ATMOS.2005.659

TÖRNQUIST, J.; Persson, J. A. N-tracked railway traffic rescheduling during disturbances. Transportation Research Part B 41(3):342-362, 2007. DOI:10.1016/j.trb.2006.06.001

TÖRNQUIST, J. N Design of an effective algorithm for fast response to the re-scheduling of railway traffic during disturbances. Transportation Research Part C vol. 20(1): 62-78, 2012. DOI:10.1016/j.trc.2010.12.004 